\title{
Validation Results of a Self-Maintained Cardiology Collaborative Tool
}

\author{
E del Hoyo-Barbolla ${ }^{1}$, E Conde $^{1}$, MT Arredondo ${ }^{1}$, E Villalba $^{1}$, \\ M Martínez-Sellés ${ }^{2}$, MI Mené ${ }^{3}$ \\ ${ }^{1}$ Life Supporting Technologies, Madrid, Spain \\ ${ }^{2}$ Hospital General Universitario Gregorio Marañón Madrid, Spain \\ ${ }^{3}$ Medtronic, Madrid, Spain
}

\begin{abstract}
Excellence in care optimisation in modern cardiology is a twofold issue that involves both the collaboration of numerous communities that benefit from collaborative work spaces as well as life-long education of professionals. Progressive specialisation of medical professionals is one of the factors that most account for this lack of dissemination giving way to the creation of clustered and specialised knowledge domains, only accessible to the elite of specialists and professional networks created around them. Besides, communication and collaboration among professionals proves to be quite scarce and hard outside restricted circles.

This paper presents the results and lessons learnt from Health Memory (HM), a self-maintained multidisciplinary system that focuses on cardiology. It aims at enabling healthcare professionals (GPs, cardiologists,other personnel,etc.), to address the management of knowledge and the enhancement of communication mechanisms.
\end{abstract}

\section{Introduction}

Excellence in care optimisation in modern cardiology is a twofold issue that involves both the collaboration of numerous communities that benefit from collaborative work spaces as well as life-long education of professionals. Progressive specialisation of medical professionals is one of the factors that most account for this lack of dissemination giving way to the creation of clustered and specialised knowledge domains, only accessible to the elite of specialists and professional networks created around them. Besides, communication and collaboration among professionals proves to be quite scarce and hard outside restricted circles.

This paper presents the results and lessons learnt from Health Memory (HM), which is a self-maintained collaborative and multidisciplinary system that focuses on cardiology [1], [2]. It aims at enabling healthcare professionals (e.g. general practitioners, cardiologists, personnel from medical companies, etc.), to address both the sharing and improving of knowledge management and the enhancement of communication mechanisms.

The system has been thoroughly validated in two hospitals: the S. Maria degli Angeli Hospital in Pordenone (Italy) and the Hospital General Universitario Gregorio Marañón (HGM) in Madrid, Spain, in two phases: the first one focused on the assessment of system usability and users adaptation to the system services in the improvement of patients' care process. The second one focused on the knowledge management issues: sharing, ranking and retrieving information of relevant experiences using the powerful HM search engine among hospital and non-hospital cardiologists.

The evaluation of the opinion of professionals consisted of a self-evaluation with a questionnaire. The questionnaires contained closed-ended questions and respondents were provided space to add written comments, which were really valuable to advance with our research.

Preliminary results conclude that $\mathrm{HM}$ is considered a powerful tool for knowledge exchange and community building. Furthermore HM is viewed as a complementary search tool to specialised on-line libraries (external search provides both a set of documents filtered by HON [3] tools and Pub Med articles) presenting more concrete results, due to the Cardiontology [4], [5] which makes the search engine very powerful. Besides, the possibility of ranking and exchanging personal comments on published knowledge (e.g. papers, guidelines, etc.), sharing routine experiences (i.e. clinical cases, forum threads, etc) and having a repository for non-publishable knowledge, are considered extremely valuable.

Health Memory allows cardiologists to create virtual communities that enable the sharing of knowledge, thus improving their competence, by facilitating continuity of care for patients and by taking evidence-based decisions at the point of care. 


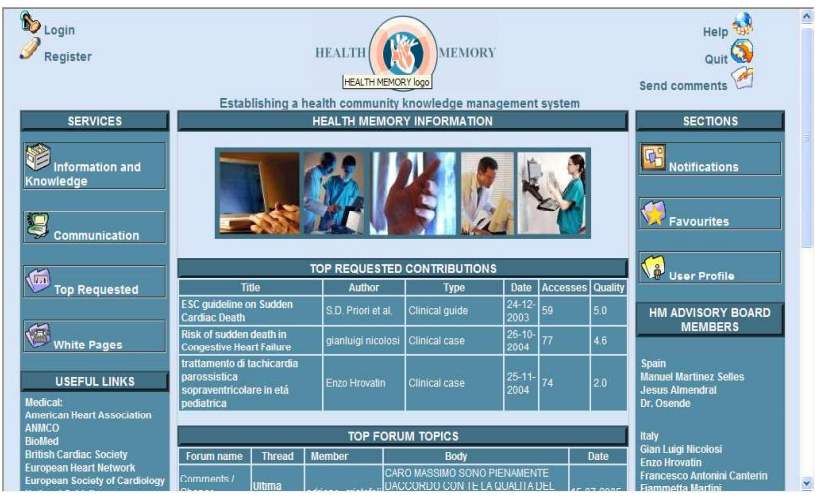

Figure 1. HM System appearance

\section{Methods}

The core of HM is a web portal that provides the user, by means of an intuitive graphical user interface (GUI) access to the different built-in functionalities, grouped into services. In the back-end, an agent platform enables the convenient processing of all the knowledge managed by the system. A newly developed ontology, focused on the field of cardiology, provides a powerful search engine that enhances the knowledge search and communication processes.

Medical professionals have tested the system in numerous ways and at different levels and an evaluation of the system has been already carried out. This validation and evaluation process has been developed in different stages at the hospitals previously mentioned. This article will focus on the evaluation process that took place in the Spanish Hosptial.

The evaluation took place by organising different meetings with the specialists in the Gregorio Marañón Hospital in Madrid. A first meeting was held in Madrid (HGM) where the Health Memory prototype was shown to non-hospital Cardiologists who participated in the Health Memory validation. In this session the system was presented and opinions and comments where registered although no qualitative or quantitative formal evaluation was performed. Lately, a second meeting was held in Madrid where the Health Memory prototype was demonstrated to general physicians (GP, or in Spanish "medico de familia") who participated in the first Health Memory validation and evaluation. Finally, on a third meeting was held with non-hospital Cardiologists who had already participated in the first meeting in order to perform a formal and recorded system validation and evaluation. The last phase took place with these nonhospital cardiologists in which the users introduced clinical cases in the system and evaluated this functionality.

\section{Results}

\subsection{Evaluation with GPs}

After a first explanation of what HM was, how the system worked and its main services and functionalities, participants were shown how to reach the system (via the www.health-memory.org URL) and register. A first problem encountered was that the system had not been designed to cope with a high number of registrations at the same time (probably due to the number of accesses to the remote database, physically situated in Greece).

Most of the GP who were of a young age showed a medium to high IT literacy and some of them provided a high number of suggestions that were taken into account in order to redesign some of the GUIs and the services.

Their main interest and concern was on understanding and getting to use the tools that would provide them communication with other GP and mainly with the specialists, which they found difficult to get hold of on a daily basis. Furthermore, they were also keen on retrieving up to date information on new recommendations (guidelines, clinical protocols, etc).

Questionnaires were handed out after the session and they were easily used and completed. The following figures show the results obtained from these questionnaires.

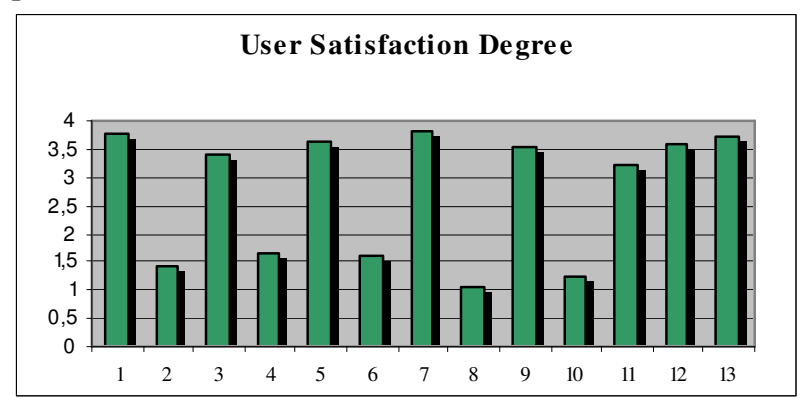

Where the questions numbers stand for:

1. I'd like to use this system frequently

2. I found the system unnecessarily complex (reversed)

3. The system was easy to use

4. I'd need the support of a technical person to use it (reversed)

5. HM functions were well integrated

6. There were inconsistencies (reversed)

7. It is easy to learn the system quickly

8. The system was cumbersome to use (reversed)

9. I felt confident using the system

10. I need to learn many things before using HM (reversed)

11. Uploading knowledge is easy and effective

12. Searching and retrieving knowledge is easy

13. The features offered by HM are user friendly

Table 1. User satisfaction degree. 
Note that questions 2, 4, 6, 8 and 10 are reversed, so from the figure it can be argued that the user satisfaction degree with the system is quite high.

The system capability is also positively valued. The ranking for this questions was $1=$ no and $2=y e s$. As questions are "positive" and closer to 2 than to 1 , we understand the valuation is quite positive.

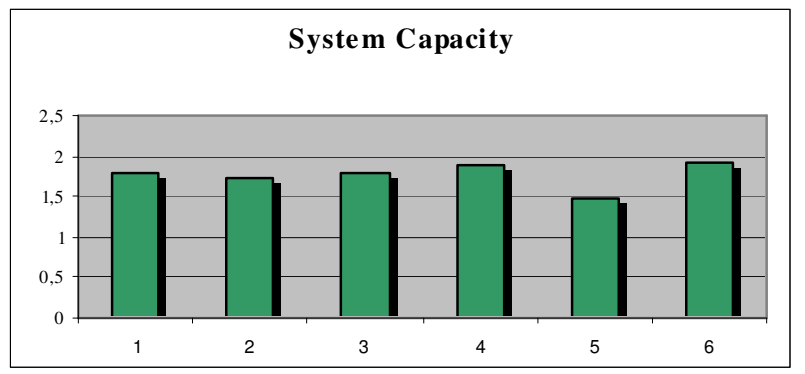

Where the questions numbers stand for:

1. Exploring by trial and error

2. System reliability

3. Are use actions required consistent?

4. Performing tasks is straightforward

5. HM has the capabilities I expect it to have

6. Is board of experts reliable and qualified?

Table 2. System capacity.

The system usefulness is, in general also positively valuated. However a high percentage entended to continue using other information sources more frequently than HM. Questions 6 and 9 are asked in the "reversed" form.

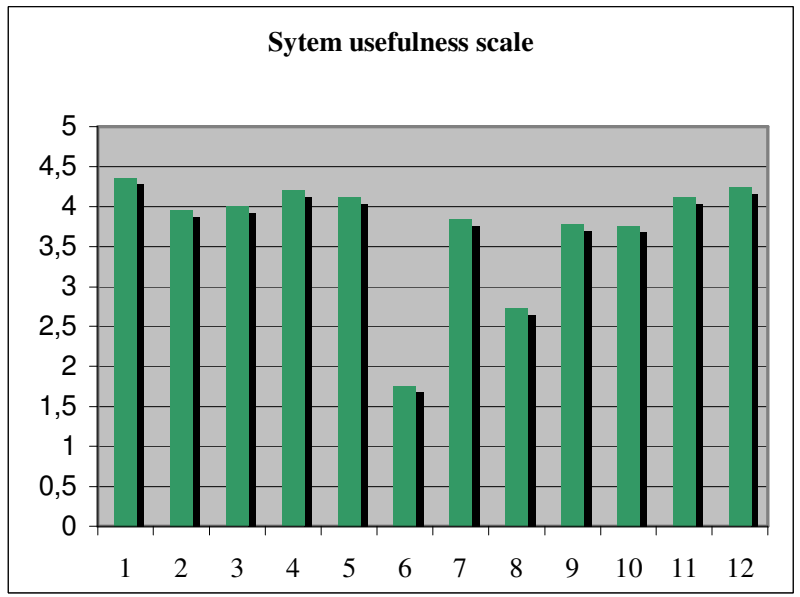

Where the questions numbers stand for:

1. HM will help me improve my knowledge

2. HM will support my diagnostic decisions

3. HM will support my therapeutic decisions
4. 24/7 availability will help my effectiveness

5. HM will improve the communication with my colleagues

6 . The system will not improve my effectiveness

7. HM gives visibility on cardiology research and practice

8. I think HM is better than other existing bibliography sites (PubMed, AHA, etc)

9. I'd rather use other information sources

10. I'll use HM as a basis for researches

11. HM community support tools can improve daily practice

12. HM should be integrated with other services and EHRs

Table 3. System usefulness.

\subsection{Evaluation with non-hospital cardiologists}

The third session involved non-hospital cardiologists in influence area of Hospital Gregorio Marañón in Madrid.

The lack of IT skills in this group (apart from an advanced user, from whom extremely interesting comments were made) proved hard to overcome. In this sense we recommend that prior classification (according to IT literacy) can be done so as to target the evaluation towards different kind of indicators and comments (i.e. more in depth functionality and appearance issues with advanced users and more "orientation" concerns with less skilled users).

The overall impression on Health Memory objective is extremely useful, in particular the possibility to establish targeted searches, classification of specific contributions as well as an easy horizontal and/or vertical (as desired) communication (cardiologist-cardiologists, GP-GPs, or GP with cardiologists or vice-versa) among colleagues, which was really appreciated.

The user-friendliness, accessibility and organisation of the web portal pages and the different services were examined and although many suggestions emerged to improve navigation and orientation among the different services in the first version (evaluated version), the general comment was that the new version improved the accessibility and the orientation of users when navigating the site.

The "Send" functionality is useful

$86,67 \%$

I would suggest other professionals the use of HM to upload content

I like the result of clinical cases

$80 \%$

I would add other fields to the

clinical case

$80 \%$

The structure eases the elaboration

of clinical cases

$86,67 \%$

I spend a similar time to that 
expected

HM provides me the documents

expected

$50 \%$

HM provides me quality documents

$43 \%$

The list does not contain irrelevant

documents

$57 \%$

Table 4. Results from the individual session validation

The fourth stage of the evaluation phase consisted on individual sessions with the cardiologists in which they introduced clinical cases and evaluated this functionality as well as the searching of documents. From all the group that tool part in the previous phase, only 7 cardiologists moved on to this stage. Table 4 shows the results obtained from this evaluation.

\section{Discussion and conclusions}

Modern cardiology is increasingly growing in complexity. Scientific advances lead to the development of new diagnosis, treatment and management solutions, providing healthcare professionals with a great amount of information that many times results very difficult to handle, or even remains hidden. In this context, the development of knowledge search and management systems, which facilitate communication among different cardiology areas, reveals to be an important factor.

The Health Memory system allows the creation of a virtual community in the cardiology domain which enables professionals to share and increase knowledge, as well as to improve their own specific competence, facilitating continuity of care for patients and the taking of evidence-based decisions at the point of need.
This work has been performed in the framework of the IST project Health Memory (IST-2001-38181), which is partly funded by the European Commission.

\section{References}

[1] The Apache Struts Web Application Framework. http://struts.apache.org/.

[2] Bellifemine F, Caire G, Poggi A, Rimassa JADE: A White Paper. Exp in search of innovation. Volume $4-\mathrm{n} .1-$ March 2004. Telecom Italia Lab.

[3] Health on the Net Foundation. http://www.hon.ch/.

[4] Fernández M., Gómez-Pérez A. Juristo N. Methonthology: From Ontological Art Toward Ontological Engineering. Spring Symposium Series on Ontological Engineering. AAAI97. Stanford. USA. March 1997.

[5] Noy NF, Sintek M, Decker S, Crubezy M, Fergerson RW, Musen MA. Creating Semantic Web Contents with Protege-2000. IEEE Intelligent Systems 16(2):60-71, 2001.

Address for correspondence

Eva del Hoyo-Barbolla

Life Supporting Technologies, ETSI Telecomunicación, Ciudad Universitaria s/n., Madrid 28040.

e-mail: evahb@1st.tfo.upm.es

Tel. +34915495700 ext 332

Fax:+34913366828

\section{Acknowledgements}

